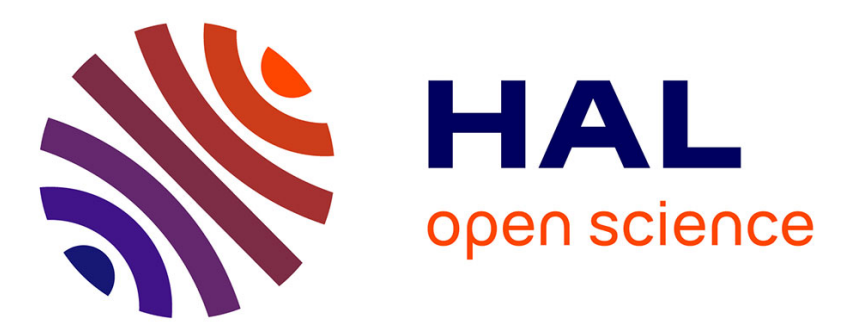

\title{
Mitigating the impact of GNSS signals in the radio astronomy band 1610.6-1613.8 $\mathrm{MHz}$
}

Olivier Julien, Jean-Luc Issler

\section{To cite this version:}

Olivier Julien, Jean-Luc Issler. Mitigating the impact of GNSS signals in the radio astronomy band 1610.6-1613.8 MHz. ION GNSS 2008, 21st International Technical Meeting of the Satellite Division of The Institute of Navigation, Sep 2008, Savannah, United States. pp 921-932. hal-01022448

\section{HAL Id: hal-01022448 \\ https://hal-enac.archives-ouvertes.fr/hal-01022448}

Submitted on 30 Sep 2014

HAL is a multi-disciplinary open access archive for the deposit and dissemination of scientific research documents, whether they are published or not. The documents may come from teaching and research institutions in France or abroad, or from public or private research centers.
L'archive ouverte pluridisciplinaire HAL, est destinée au dépôt et à la diffusion de documents scientifiques de niveau recherche, publiés ou non, émanant des établissements d'enseignement et de recherche français ou étrangers, des laboratoires publics ou privés. 


\title{
Mitigating the Impact of GNSS Signals in the Radio Astronomy Band 1610.6-1613.8 MHz
}

\author{
Olivier Julien, Ecole Nationale de l'Aviation Civile, Toulouse, France \\ Jean-Luc Issler, Centre Nationale d'Etudes Spatiales, Toulouse, France
}

\section{BIOGRAPHIES}

Olivier Julien is an assistant professor at the signal processing laboratory of ENAC (Ecole Nationale de l'Aviation Civile), Toulouse, France. His research interests are GNSS receiver design, GNSS multipath and interference mitigation and GNSS interoperability. He received his B.Eng in 2001 in digital communications from ENAC and his PhD in 2005 from the Department of Geomatics Engineering of the University of Calgary, Canada.

Jean-Luc Issler is head of the Transmission Techniques and signal processing department of CNES, whose main tasks are signal processing, air interfaces and equipments in Radionavigation, TT\&C, propagation and spectrum survey. He is involved in the development of several spaceborne receivers in Europe, as well as in studies on the European RadioNavigation projects, like GALILEO and the Pseudolite Network. With DRAST, he represents France in the GALILEO Signal Task Force of the European Commission. He received in 2004 the Astronautic Prize of the AAAF (French aeronautical and space association) and in 2008 the EADS science and engineering prize of French Academy of Science for his technical work on GALILEO signals and spaceborne GNSS equipments.

\section{ABSTRACT \\ The band $1610.6-1613.8 \mathrm{MHz}$ is used by the radio astronomy (RA) community in order to study the spectral line of the Hydroxyl $(\mathrm{OH})$ radical transitions. This radical is considered to be one of the most important lines for the observation of physical phenomena associated with the formation of protostars and the initial stages of star formation. The current ITU recommendation to protect this RA band is to ensure an Equivalent Power-Flux Density (EPFD) lower than $-258 \mathrm{dBW} / \mathrm{m}^{2} / 20 \mathrm{kHz}$.}

Radio Frequency Interference (RFI) has, however, been reported in this band that significantly disturbs the exploitation of $\mathrm{OH}$ spectral lines. Examples of these interference are GLONASS L1 signal and Iridium
[Monstein and Meyer, 2007; ERC, 1997; Galt, 1991]. In order to be immunized from these sources of disturbance, different antenna or signal processing techniques have been investigated and used by the RA community. Cooperation with GLONASS was also used in order to reach an agreement satisfying both parties.

The main reason for GLONASS to provide unwanted interference in the RA band is its close proximity with this band. Although further away from the RA band than GLONASS, Galileo is still relatively close to it $(15 \mathrm{MHz}$ only). In particular, the ITU recommendation on the RA band implies a fairly steep slope to the Galileo E1 payload filter, which represents a high constraint for the realization of the payload. Consequently, it might result in imperfect filtering of the signals, potentially leaking in the RA band.

It is then interesting to develop a method that could mitigate the effect of GNSS signal in the RA band, in order to ensure that after using this method, the level of the interference PFD is below the ITU recommendation. This is the goal of this paper, which introduces a general method to mitigate GNSS signals (using Code Division Multiple Access (CDMA) technique) from the RA band. In particular, the method is adapted to signals that have an open signal and a restricted signal, with is generally the case for GNSS signals.

The method is based on the estimation of the GNSS signal, followed by its subtraction from the incoming signal in order to just leave the RA signal of interest. The paper particularly emphasis the adaptation of this method to the Galileo E1 signal, showing a reduction of approximately $10 \mathrm{~dB}$ of the maximum level of the expected Galileo PFD within the RA band.

\section{INTRODUCTION}

The radio-astronomy (RA) band $1610.6-1613.8 \mathrm{MHz}$ is an important RA band since it allows studying Hydroxyl $(\mathrm{OH})$ radical transitions that are necessary to observe Maser effects in certain star forming regions, in giant red stars and in comets. In order to protect this band from significant radio-frequency (RF) pollution, the 
International Telecommunication Union (ITU) issued Resolution 739 for systems using adjacent bands.

Before the adoption of this resolution and despite the existing ITU-R recommendations, there have been numerous records of strong interference in the RA band of interest over the past decades. Examples of these recorded interference are GLONASS L1 signals and Iridium [Monstein and Meyer, 2007; ERC, 1997; Galt, 1991].

The case of the GPS L1, Galileo E1 and GLONASS G1 bands are of interest since they are all located just below the considered RA band. In particular, there are specific ITU recommendations targeting Radio-Navigation Satellite Systems (RNSS) to protect the RA band. These recommendations are particularly strict for RNSSs that have a significant part of the useful signal located very close to the RA band. This is the case for GLONASS G1 signals and for Galileo E1 signals.

The ITU resolution on the RA band implies a high constraint on the satellite payload, in particular considering that the realization of good filters with a high rejection of adjacent bands is often a difficult task for satellite use.

Besides, it has to be kept in mind that the ITU issued a resolution including only a consultation process when a given threshold is exceeded, which means that it is not mandatory to respect this threshold per say. If the threshold is not respected by a specific system, it is mandatory that the representatives of the system engage a consultation process with the RA community, represented by the Scientific Committee on Frequency Allocation for Radio Astronomy and Space Science (IUCAF).

This is what happened with GLONASS, and discussions started with the IUCAF in 1983. After 10 years of coordination, several agreements were reached [Cohen, 1993] and implemented by GLONASS. More recently, at the World Radiocommunication Conference (WRC) 2007, GLONASS was excluded from the ITU resolution concerning the RNSS, leaving the protection of the RA band to the bilateral Russian Federation/IUCAF agreements [Ohishi, 2007].

Based on this background, it is important to ensure the protection of the RA band despite the tight ITU conditions.

The main goal of this paper is then to present and assess a new processing technique that enables mitigating the impact of any RNSS signal using CDMA on the RA signal of interest by estimating the RNSS signal before subtracting it from the useful RA signal. This could represent an interesting technique in case an RNSS does not fulfill completely the ITU recommendations.

The article will first introduce the ITU recommendations and review the main steps of the GLONASS/IUCAF agreements. Then, the Galileo E1 signal will be introduced and the constraints put by the ITU recommendations for the RA band 1610.6-1613.8 MHz on the Galileo E1 payload will then be investigated. Finally, the proposed mitigation algorithm will be reviewed, explained and analyzed in the Galileo context. In particular, the algorithm will be tested and its initial implications on the Galileo payload filter will be stated.

\section{ITU RECOMMENDATIONS ON RA BAND 1610.6- 1613.8 MHz}

The observation of the $\mathrm{OH}$ radical transition realized around $1612 \mathrm{MHz}$ is very sensitive to the interference environment present in the band since the investigated signal is received with a very low power. RA is a primary user of this band and thus, to protect the RA observations, the ITU has made several recommendations, including some dedicated to RNSS systems:

- Recommendation ITU-R RA.1513 indicates that the percentage of data loss due to interference from one single system should not exceed $2 \%$ at any RA station. The percentage of data loss is defined as the percentage of integration periods of 2000s in which the average aggregate spectral Power Flux Density (PFD) at the radio telescope exceeds the level given in the resolution ITU-R739.

- For Non-Geo-Stationary Orbit (NGSO) satellite systems, such as Galileo, the resolution ITU-R 739 considers the maximum Equivalent PFD (EPFD) level to be $-258 \mathrm{dBW} / \mathrm{m}^{2} / 20 \mathrm{KHz}$ for a single dish antenna.

- Recommendation ITU-R M.1583 provides a methodology to evaluate the level of emissions (expressed in terms of EPFD) of a NGSO satellite system at the RA site:

- The sky is divided into cells of nearly equal solid angle

- A statistical analysis is performed with 2 random variables:

- The direction of the pointing antenna

- The starting time of the satellite constellation

- For each trial, the unwanted emission level is averaged over 2000s.

- ITU-R RA.1631 provides the antenna pattern and maximum antenna gain to be used in compatibility studies involving the RA. RA sites use parabolic antennas with a typical diameter between 13 and 100 meters. Such radio-telescopes provide a highly directional antenna with low side-lobes. Typical antenna gain patterns are reproduced in Figure 1. It can be seen that, for the range of antenna considered (13- to 100-meter diameter), the main lobe of the 
antenna is very sharp with a very high maximum gain (47 to $64 \mathrm{dBi}$ ).
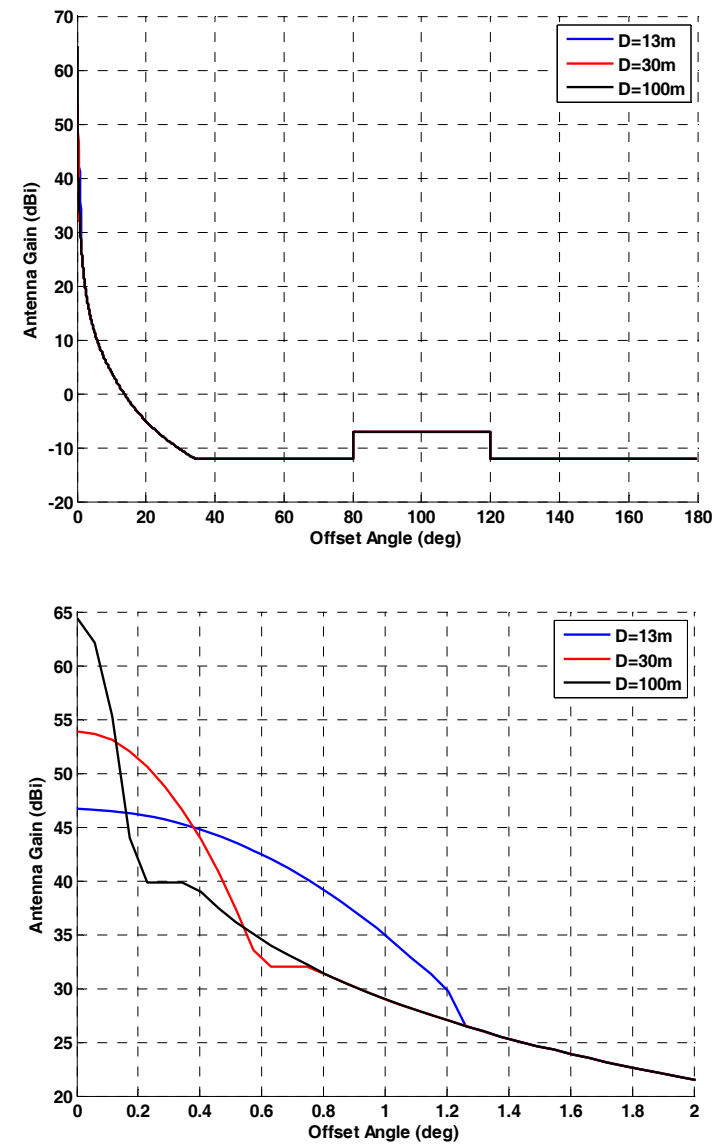

Figure 1 -Antenna Gain Pattern of a Typical RadioTelescope (Top) and Exploded View (Bottom)

\section{The CASE OF GLONASS}

The case of GLONASS is interesting since it is historically the first example of an RNSS system that was recognized as not respecting the ITU recommendation.

The main reason for this is the close proximity of the GLONASS band with respect to the RA band. Indeed, GLONASS uses an FDMA modulation with carrier frequencies that were originally located at $1602 \mathrm{MHz}+$ $\mathrm{k} \times 562.5 \mathrm{KHz}$ with $\mathrm{k} \in[0 \ldots 21]$. It can be noticed that the channels 16 to 21 fall directly in the RA band.

An example of such interference is shown in Figure 2, taken from [Galt, 1991]. It can clearly be seen that the GLONASS signal is heavily present in the RA band and will interfere with the observation of the $\mathrm{OH}$ transition.

The recognition, by the RA community, of the problem paused by GLONASS had 2 main impacts:

- The initiation in 1983 (one year after the first GLONASS satellite was launched) of a coordination process between the IUCAF and the Russian Federation in view of accepting a series of agreements to reduce the constraints on GLONASS, while allowing acceptable levels to the RA community.

- The development by the RA community of advanced techniques to mitigate these interference.

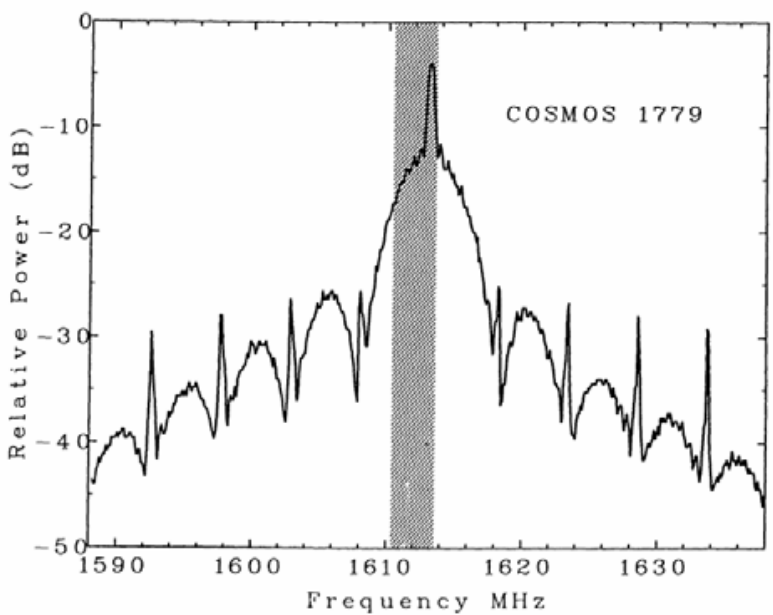

Figure 2 - PSD of an Apparent GLONASS Interference Located within the RA Band [Galt, 1991]

The collaboration between the IUCAF and the Russian Federation started with the realization of a set of experiments designed to test different configurations of the GLONASS frequency plan. Based on the results, a set of recommendations were reported by the WG7 of the Radiocommunication Section. The recommendations mostly suggested that GLONASS should slowly reassign its frequency channels to the lower channels. Besides, it is also suggested that GLONASS should try to filter the high frequency part of its signals. Finally, on Nov. $4^{\text {th }}$ 1993, an official agreement was signed, with its conclusions are reminded in [Cohen, 1993]. The main points of the WG7 of the Radiocommunication Section were greatly re-stated with an emphasis on the continuation of GLONASS/IUCAF collaboration.

Currently, GLONASS reassigned its frequencies to channels -7 to 6 , directly reducing the amount of unwanted emission in the RA band 1610.6-1613.8 MHz. Still the level of emission is greater than the initial ITU-R RA.769 (by approximately $12 \mathrm{dBs}$ for a single satellite when using a central frequency close to the RA band).

Recent GLONASS satellites have incorporated a notch filter in order to reduce the satellite emission in the RA band. This is clearly represented in Figure 3Figure 3, taken in 2005 from Leeheim for the satellite COSMOS 2411. In this figure, the considered GLONASS satellite has a central frequency of $1604.25 \mathrm{MHz}$. 


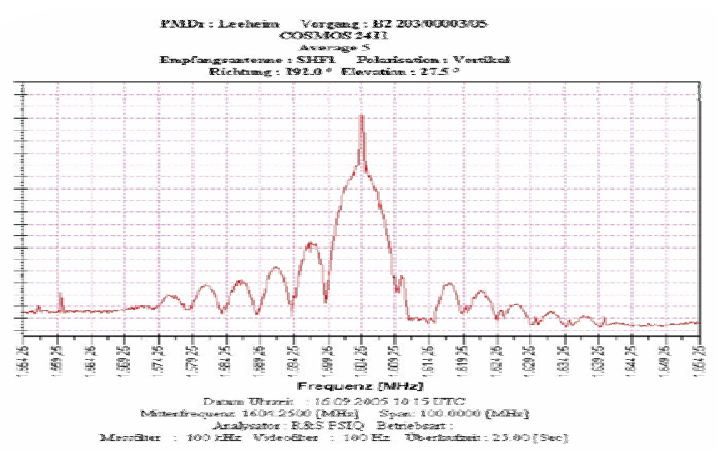

Figure 3 - PSD of an Observed GLONASS Signal

Beside the collaboration initiative, there have been many publications by the RA community on interference removal (not restricted to GLONASS interference). Several antenna and/or signal processing techniques have been investigated and used with success [Bower, Brings, 2000; Bunton, 2001; Jeffs, 2005; Leshem, 2000; Poulsen, 2004].

In particular, specific techniques have been designed to mitigate the GLONASS C/A disturbances. One of them consisted in tracking accurately the GLONASS signal, using the high gain radio-telescope, in order for the RA receiver to estimate the signal's amplitude, code delay and phase. This meant that the GLONASS C/A signal could then be reproduced accurately (the spreading sequence is known) and subtracted from the incoming signal of interest. This process is described in Ellingson et al. (2001).

In the case of the GLONASS P signal, the results were not as successful since only authorized receivers can track the encrypted code. Some estimation techniques were tried [Bunton, 2001], but with the drawback of increasing the background noise.

\section{The Galileo Case}

\section{a. Galileo E1 Signal Structure}

The Galileo E1 signal will be broadcast at a central frequency of $1575.42 \mathrm{MHz}$ and within its allocated transmission bandwidth of $40.92 \mathrm{MHz}$. It will support 2 Galileo services:

- The OS that is accessible to any user that wants to receive Galileo signals. Two signals, known as OSA and OSB, are supporting this service:

$\circ$ The OSA signal is also referred to as the OS data component and carries the OS navigation message at a rate of $250 \mathrm{symbol} / \mathrm{sec}$.

o The OSB signal is also referred to as the OS pilot component and is just a ranging signal not modulated by any navigation message.
Both components are synchronized. They are both modulated by spreading codes of length 4092 with a rate of $1.023 \mathrm{Mchips} / \mathrm{sec}$. The OSB also uses a secondary code of length 25 at a rate of 250 chips/s. The overall OS signal uses a $\operatorname{CBOC}(6,1,1 / 11)$ modulation, described in Hein et al (2007).

- The PRS that is restricted to authorized users such as public safety or emergency services. Only one signal is used on the Galileo E1 band to support this service. It uses a cosine-phased $\operatorname{BOC}(15,2.5)$ modulation. The spreading code used is encrypted and aperiodic.

The theoretical expression of the transmitted unfiltered Galileo E1 signal is given in [Rebeyrol 2007] by:

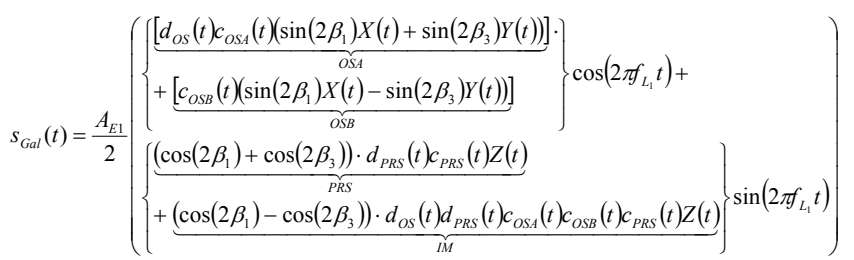

where

- $A_{E 1}=\sqrt{2 P_{E 1}}$ is the amplitude of the overall Galileo E1 signal,

- $P_{E 1}$ is the power of the overall Galileo E1 signal,

- $d_{O S A}$ and $d_{P R S}$ are the navigation messages carried by the OS and PRS signals,

- $c_{O S A}, c_{O S B}$ and $c_{P R S}$ are the spreading sequences carried by the OS and PRS signals (note that $c_{O S B}$ actually contains a primary and a secondary code),

- $X, Y$ and $Z$ represent respectively the sinephased $\operatorname{BOC}(1,1)$, the sine- phased $\operatorname{BOC}(6,1)$ and the cosine-phase $\mathrm{BOC}(15,2.5)$ sub-carriers,

- the coefficients $\beta_{1}$ and $\beta_{3}$ are a function of the relative power of each component of the Galileo E1 signal. Currently, the respective power of the PRS signal with respect to the other signals is not publicly available, and

- $f_{L_{1}}$ is the $\mathrm{L} 1$ carrier frequency (1575.42 MHz).

It can be noticed in the previous equation that the fourth term does not represent any of the useful signals. It is known as the Inter-Modulation (IM) signal and it is used to create a signal with a constant envelope, so that the High Power Amplifier within the satellite payload can be used optimally. As it can be seen, it is a mix of the PRS and the OS components. It has a cosine-phased $\operatorname{BOC}(15,2.5)$ modulation.

As already mentioned, the relative power of each component is not fully known yet and thus only 
assumptions can be made to determine the value of the parameters $\beta_{1}$ and $\beta_{3}$. In this paper, it will be assumed that the PRS signal has the same power as the OS signal. This assumption leads to:

$\beta_{1}=0.5788858 ; \quad \beta_{3}=0.1469235$

Assuming uncorrelated spreading codes between components, it can then be deduced that the Galileo E1 normalized (and unfiltered) Power Spectral Density (PSD) is:

$$
S_{G a l}(f)=\left(\begin{array}{l}
\frac{\sin ^{2}\left(2 \beta_{1}\right)}{2} \cdot S_{s B O C(1,1)}(f)+\frac{\sin ^{2}\left(2 \beta_{3}\right)}{2} \cdot S_{s B O C(6,1)}(f) \\
+\frac{\cos ^{2}\left(2 \beta_{1}\right)+\cos ^{2}\left(2 \beta_{3}\right)}{2} S_{C B O C(15,2.5)}(f)
\end{array}\right)
$$

The shape of each individual PSD can be found in Rebeyrol (2007).

Before being transmitted, the Galileo E1 signal is filtered by the Galileo satellite payload so that it complies with its transmitted bandwidth assignment. This filtering also intends to reduce the level of interference in adjacent bands.

\section{b. Galileo E1 and the Radio Astronomy Band 1610.6-1613.8 MHz}

It is thus important to ensure that Galileo E1 will meet the previously mentioned ITU recommendations in order not to harm the RA users. Figure 4 (top) shows the unfiltered Galileo E1 PSD with respect to the considered RA band. Two important facts can be noted in Figure 4:

- The Galileo E1 transmitted band ends very close from the PRS side-lobes. This means that the filtering done in the Galileo E1 payload to limit the transmitted band has to be carefully done in order not to deteriorate the PRS signal and thus the user.

- There is a far side-lobe of the Galileo E1 signal in the middle of the RA band.

Figure 4 (bottom) zooms on the normalized (and unfiltered) PSD of the Galileo E1 signal (and its different components) within the RA band. It can be seen that it is highly dominated by far secondary side-lobes of the PRS and IM signals. This means that it is mainly the PRS component that might affect the RA band. Comparatively, the PSD of the $\mathrm{BOC}(1,1)$ component is more than $5 \mathrm{dBs}$ below, while the PSD of the $\operatorname{BOC}(6,1)$ component is more than $15 \mathrm{~dB}$ below.

Based on the ITU recommendations, the Agence Nationale des FRequences (ANFR) wrote a program in order to compute the maximum PFD per Galileo satellite, following the methodology ITU-R. M.1583. A description of the program is given in [ANFR]. The following settings were selected:

- A constellation of 27 satellites,

- A 100-metre diameter radio-telescope located in Effelsberg $\left(\mathrm{Lat}=50.7^{\circ}\right.$; Long $\left.=7^{\circ}\right)$ in Germany,

- The sky is split in 2334 cells of 9 square degrees.
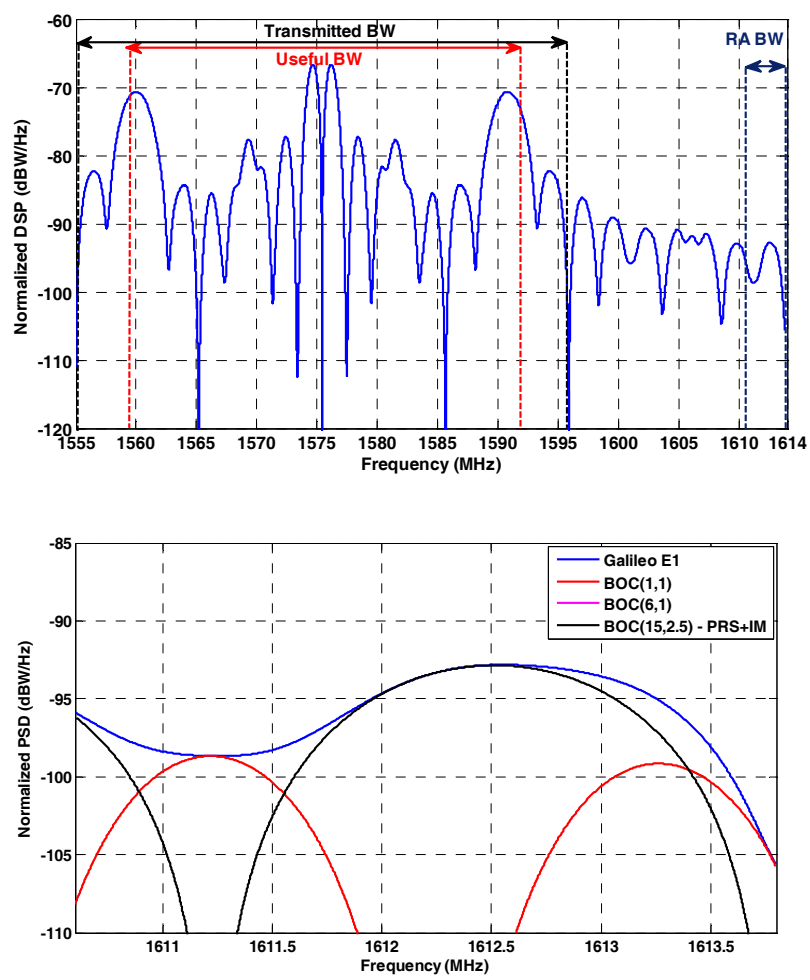

Figure 4 - Unfiltered Galileo E1 PSD in E1 Band and RA Band (Top) and Zoom on the RA Band (Bottom)

Using this configuration, the maximum authorized PFD per satellite to ensure the recommendation ITU-R M.1583 was assessed to be $-212 \mathrm{dBW} / \mathrm{m}^{2} / 20 \mathrm{kHz}$ in the RA band.

According to [Galileo OS ICD], the maximum received power for the Galileo E1 OS component at the output of a $0 \mathrm{dBi}$ antenna is $-154 \mathrm{dBW}$. Following our assumptions, this means that the maximum received E1 power from 1 Galileo satellite is approximately $-150.64 \mathrm{dBW}$.

The ratio between the total unfiltered Galileo E1 signal and the power contained in a $20 \mathrm{KHz}$ bandwidth is plotted in Figure 5 for the considered RA band. It can be seen that the maximum value is $-49.8 \mathrm{~dB}$.

Moreover, the effective area of a $0 \mathrm{dBi}$ antenna at $1593.834 \mathrm{MHz}$ (center of the PRS side-lobe that is close to the RA band) is equal to $25.5 \mathrm{~dB} \cdot \mathrm{m}^{2}$ [Lestarquit, 2000]. 
Consequently, the unfiltered PFD of a single Galileo satellite in the RA band equals $-150.64-49.8+25.5=-$ $174.94 \mathrm{~dB} / \mathrm{m}^{2} / 20 \mathrm{KHz}$.

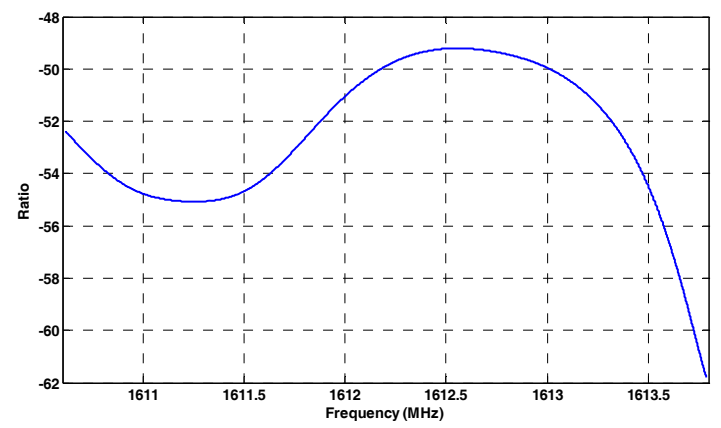

\section{Figure 5 - Ratio between the power contained within the transmitted bandwidth and the power contained in a $20 \mathrm{KHz}$ band in the RA band}

This means that the required attenuation provided by the payload filter in the RA band should be equal to $174.94+212=37.1 \mathrm{dBs}$ in order to meet the ITU recommendations.

Such a rejection (37.1 dBs in $15 \mathrm{MHz}$ ) implies a high constraint on the Galileo payload filter. This is particularly true since the PRS signal that has its main lobes at the edge of the Galileo E1 transmitted band.

It would thus be interesting to find a way to reduce this payload filter constraint for Galileo without harming the RA community.

\section{Proposed Strategy to Reduce the Impact of RNSS on the RA Band}

\section{a. Overview of the Proposed Technique}

The idea behind the proposed method is to take advantage of the high radio-telescope antenna gain to try to estimate each chip of the Galileo PRS signal. Assuming that behind the radio-telescope, there is a wide-band Low Noise Amplifier (LNA), the signal can then be split in 2:

- A channel that leads to a GNSS processing block which goal is the estimation of the Galileo E1 baseband signal.

- A channel that leads to the RA signal processing block. This block should also have, as input, the estimated Galileo E1 signal.

The GNSS processing block can be activated when a Galileo satellite is entering the second or third side-lobe of the radio-telescope gain pattern. This can be done either through signal processing/detection, or through the simple use of the satellites ephemeris.

At this stage, given the sharpness of the radio-telescope gain pattern, it seems appropriate to consider that only 1
GNSS satellite will be present in the main lobe. The probability of having several satellites from different GNSS within the first two lobes of the radio-telescope has to be assessed, but is assumed very low.

Also the method is suited for GNSS signals that have synchronized open and authorized (unknown spreading sequence) components.

The proposed algorithm has three steps:

- The first step of the GNSS processing block is to get synchronized with the signal of interest. This means implementing a method similar to Ellingson et al. (2001). This should be easily achieved using the open component of the GNSS signal, especially considering the high gain of the parabolic antenna. This synchronization has several objectives: (1) since the different components of the signal are usually synchronized, it also provides synchronization with the restricted signal; (2) a fine estimation of the open signal characteristics will also provide information on the restricted signal characteristics (such as amplitude).

- The second step of the GNSS processing block is to estimate the value of each samples of the restricted signal based on estimation theory.

- The third step is to transform the estimated GNSS baseband signal (a succession of binary values for each component) into the signal perturbing the RA receiver. This means passing the estimating signal through a filter equivalent to the satellite payload filter and the receiver filter. The resulting signal is then subtracted to the signal entering the RA processing block.

Note that to achieve good results, it is tremendously important for the RA block and the GNSS to be synchronized. Thus it is ideal if the same oscillator could be used for both blocks.

\section{b. Application to the Galileo E1 Signal}

Because RA observes very low-power signals, the RA reception chain is of very high quality and made to minimize the noise factor. In the following, it will be assumed that the thermal noise PSD level at the entrance of the GNSS block equals $-205 \mathrm{dBW} / \mathrm{Hz}$.

Moreover, in the case of Galileo E1, the GNSS processing block will need a wide equivalent front-end filter in order to accept the PRS component. Thus, it is assumed that the equivalent front-end filter of this block has a one-sided bandwidth $\mathrm{B}$ of $17.9 \mathrm{MHz}$ (although it could also be interesting to look only at the side-lobe present in the RA itself, this option is not considered herein). This means that the noise power at the GNSS signal processing block input will be $-132.5 \mathrm{dBW}$. 
The assumed maximum received Galileo E1 OS signal power equals $-154 \mathrm{dBW}$. In the following, the case of a 13-meter radio-telescope will be taken. Extension to an other radio-telescope diameter is straight forward, based on the recommendation ITU-R RA.1631 for the antenna gain pattern. As already mentioned, the maximum gain of a 13-m diameter parabolic antenna is approximately 47 $\mathrm{dBi}$. Thus, the received Galileo signal of interest is amplified by $47-L \mathrm{dBs}$ where $L$ represents the antenna gain with respect to the maximum antenna gain assuming. Obviously, the reception chain will bring some losses (antenna cables, quantization, etc...). However, it is difficult to quantify these at this stage, and thus they will be included in the parameter $L$.

The correlation losses affecting Galileo E1 signal, due to the front-end filtering, are assessed to be lower than 0.15 dBs.

Consequently, the minimum $\mathrm{C} / \mathrm{N}_{0}$ to consider for Galileo E1 tracking is approximately $(98-L) \mathrm{dB}-\mathrm{Hz}$. Even for high values of $L$, this means that Galileo E1 tracking will be extremely accurate. In particular it means that the power estimation should be very accurate.

Moreover, as it was seen with the theoretical expression of the Galileo E1 signal, the OS components are in quadrature with the PRS and the IM components. This means that precise carrier removal leads to the almost perfect split between the PRS+IM and the OS signal. It is then easy to isolate the PRS from the OS.

\section{c. Estimating the Galileo E1 PRS+IM Signal}

After carrier removal and low-pass filtering, the PRS+IM component, read on the quadrature component, should be:

$Q(t)=\left[\begin{array}{l}\frac{A_{E 1} \sqrt{L_{F-B O C_{\cos }(15,2.5)}}}{4} d_{P R S}(t) c_{P R S}(t) Z(t) \times \\ \left(\begin{array}{l}\left(\cos \left(2 \beta_{1}\right)+\cos \left(2 \beta_{3}\right)\right)+ \\ \left(\left(\cos \left(2 \beta_{1}\right)-\cos \left(2 \beta_{3}\right)\right) \cdot W(t)\right\}\end{array}\right)\end{array}\right]$

where

$L_{F-B O C_{\text {cos }}(15,2.5)}$ represents the filtering losses due to the front-end filter. With a one-sided bandwidth of $17.9 \mathrm{MHz}$, the loss is approximately $1.25 \mathrm{dBs}$, and

$$
W(t)=d_{O S A} c_{O S A} c_{O S B} \text {. }
$$

Note that $W$ is known fully from the OS processing. Indeed, the spreading sequences are known, and it can be assumed that data demodulation is almost error-free due to the high $\mathrm{C} / \mathrm{N}_{0}$ values.

Then,

If $W(t)=1$ :
$Q(t)=q_{1}(t)= \begin{cases}\frac{A_{E 1} \sqrt{L_{F-B O C_{\cos }(15,2.5)}} \cos \left(2 \beta_{1}\right)}{2} \text { if } d_{P R S}(t) c_{P R S}(t) Z(t)=1 \\ -\frac{A_{E 1} \sqrt{L_{F-B O C_{\cos }(15,2.5)}} \cos \left(2 \beta_{1}\right)}{2} \text { if } \quad d_{P R S}(t) c_{P R S}(t) Z(t)=-1\end{cases}$

if $W(t)=-1$ :

$Q(t)=q_{-1}(t)= \begin{cases}\frac{A_{E 1} \sqrt{L_{F-B O C_{\text {ose }}(15,2.5)}}}{2} \cos \left(2 \beta_{3}\right) & \text { if } \quad d_{P R S}(t) c_{P R S}(t) Z(t)=1 \\ -\frac{A_{E 1} \sqrt{L_{F-B O C_{\text {os }}(15,25)}}}{2} \cos \left(2 \beta_{3}\right) & \text { if } \quad d_{P R S}(t) c_{P R S}(t) Z(t)=-1\end{cases}$

Since the sign of $W(t)$ is supposed known, the sign of $d_{P R S}(t) c_{P R S}(t) Z(t)$ is directly given by the sign of $Q(t)$. Because the magnitude of $Q$ can have 2 different values, it means that the Bit Error Rate (BER) will depend upon the sign of $W(t)$.

The theoretical BER is given by:

$$
B E R=\frac{1}{2} \operatorname{erfc}\left(\sqrt{\frac{E}{2 N_{0}}}\right)
$$

With $\operatorname{erfc}(x)=\frac{2}{\sqrt{\pi}} \int_{x}^{+\infty} e^{-t^{2}} d t$ and $E=\frac{2|Q|^{2}}{B}$.

The probability that $W(t)=1$ is equal to the probability that $W(t)=-1$, thus,

$$
B E R_{\text {tot }}=\frac{1}{4}\left(\operatorname{erfc}\left(\sqrt{\frac{2\left|q_{1}\right|^{2}}{2 B N_{0}}}\right)+\operatorname{erfc}\left(\sqrt{\frac{2\left|q_{2}\right|^{2}}{2 B N_{0}}}\right)\right)
$$

The first two rows of Table 1 summarize theoretical and simulated results for the BER. The simulations were done at baseband using:

- $\mathrm{a} 4^{\text {th }}$-order Butterworth filter of width $20 \mathrm{MHz}$ (single sided) to represent the payload filter, and

- a $3^{\text {rd }}$-order Butterworth filter of width $17.9 \mathrm{MHz}$ (single sided) to represent the receiver filter

It can be seen that there is a gap between the theoretical and simulated results. This is mostly due to the filtering effect (the PRS side-lobes are very close to the filter bandwidth edge), since the theoretical result assume perfectly squared chips. It can also be noticed that for values of $L$ as small as $20 \mathrm{dBs}$, the BER starts to increase significantly.

One way to improve the method is to take advantage of the knowledge of the PRS and IM modulation. Indeed, it is known from the theoretical formula of the Galileo E1 signal that the PRS+IM signal are modulated by a cosinephased $\mathrm{BOC}(15,2.5)$. It is then interesting to force the 
estimated signal to have such a sub-carrier. In particular, it is possible, in places where the PRS code chip remains unchanged, to compare the set of estimated samples with a pure cosine-phased $\operatorname{BOC}(15,2.5)$ sub-carrier. This, for instance can be done through a correlation process. The sign of the resulting correlation value would then give the sign of the current PRS code chip. This means that it is then possible to reproduce the PRS signal (on a chip-bychip basis).

Table 1 - Improved BER for Estimating the Sign of $d_{P R S}(t) c_{P R S}(t) Z(t)$

\begin{tabular}{|c|c|c|c|c|c|c|c|c|c|c|c|}
\hline & \multicolumn{7}{|c|}{ Losses $\mathbf{L}$ (dB) for an Radio-Telescope Diameter of 13 m } \\
\cline { 2 - 10 } & $\mathbf{4 0}$ & $\mathbf{3 7 . 5}$ & $\mathbf{3 5}$ & $\mathbf{3 2 . 5}$ & $\mathbf{3 0}$ & $\mathbf{2 7 . 5}$ & $\mathbf{2 5}$ & $\mathbf{2 2 . 5}$ & $\mathbf{2 0}$ & $\mathbf{1 7 . 5}$ & $\mathbf{1 5}$ \\
\hline $\begin{array}{c}\text { Error in the } \\
\text { Estimation of the } \\
\begin{array}{c}\text { Samples before } \\
\text { correlation (\% - } \\
\text { Theoretical) }\end{array}\end{array}$ & 43.6 & 41.6 & 38.9 & 35.4 & 31.0 & 25.7 & 19.9 & 14.0 & 9.0 & 5.0 & 2.1 \\
\hline $\begin{array}{c}\text { Error in the } \\
\text { Estimation of the } \\
\text { Samples before } \\
\text { correlation (\%- } \\
\text { Simulation) }\end{array}$ & 46.2 & 44.9 & 43.1 & 41.2 & 38.4 & 35.1 & 31.0 & 26.5 & 21.8 & 17.2 & 13.3 \\
\hline $\begin{array}{c}\text { Error in the } \\
\text { Estimation of the } \\
\text { Samples after } \\
\text { correction and } \\
\text { oversampling (\% - } \\
\text { Simulation) }\end{array}$ & 35.1 & 29.2 & 23.5 & 18.6 & 11.8 & 7.6 & 4.1 & 1.5 & 0.4 & 0.0 & 0.0 \\
\hline
\end{tabular}

This is somehow equivalent to assess the sign of the PRS code chip without the sub-carrier. The results are shown in the last row of Table 1 . It can be seen that a significant improvement (of approximately $5 \mathrm{dBs}$ in terms of equivalent incoming signal power) is reached.

Since the power of the Galileo E1 PRS signal can be assessed through the OS tracking, the estimation process of the unfiltered Galileo E1 baseband signal can be considered as completed.

It is then necessary to pass this signal through a filter equivalent to the Galileo payload filter and the RA filter, to match the process RA signal. The corrective signal can then be subtracted from the signal received by the RA block.

It is now important to see the performances of the method in reducing the actual required PFD per satellite.

\section{d. Performance of the Proposed Method}

In this section, it is assumed that the Galileo E1 OS is perfectly tracked and thus it is assumed that the PRS and IM signals have been perfectly isolated. Only these 2 signals are thus investigated.
After the PRS+IM estimation, the reproduced signal (normalized with respect to the incoming Galileo E1 PRS+IM signal) at baseband can be written as:

$Q_{R}=\alpha\left(Q+\varepsilon_{Q}\right)$

where

$\hat{Q}$ is the estimated signal, at baseband, of the normalized incoming PRS+IM signal,

$\varepsilon_{Q}=\hat{Q}-Q$ represents the estimation error of the normalized PRS+IM signal, and

$\alpha$ represents the amplitude estimation error.

It is important to remember that, even though there might be errors, $\hat{Q}$ will still have a cosine-based $\operatorname{BOC}(15,2.5)$ sub-carrier. Indeed, using the correlation method, it is possible to make an error in the sign of the PRS code chip, but the sub-carrier will always be a cosine-based BOC $(15,2.5)$. Thus, if there is an estimation error, $\hat{Q}$ will just be using a different spreading code.

Let's denote

$h$ the impulse response of the true filter equivalent to the succession of the satellite payload, the 
propagation channel, and the RA front-end filter, and

$h_{2}$ the estimation of the impulse response of the true filter equivalent to the succession of the satellite payload, the propagation, and the RA front-end filter, and

After subtraction of the estimated PRS+IM signal, considered at baseband herein, the error can be written as:

$$
\begin{aligned}
& \Delta=Q * h-Q_{R} * h_{2}=Q * h-\alpha\left(Q+\varepsilon_{Q}\right) * h_{2} \\
& \Delta=Q *\left(h-\alpha h_{2}\right)-\alpha \varepsilon_{Q} * h_{2}
\end{aligned}
$$

where the operator * represents the convolution operation.

The Fourier transform of these terms equal:

$F_{\Delta}=F_{Q}\left(H-\alpha H_{2}\right)-\alpha F_{\varepsilon_{Q}} H_{2}$

where

$F_{X}$ represents the Fourier Transform of the signal X, and

$\mathrm{H}$ and $\mathrm{H}_{2}$ represent the transfer function of the filters $h$ and $h_{2}$.

Finally, the PSD of the error signal is:

$G_{\Delta}=F_{\Delta} F_{\Delta}^{*}=\left(F_{Q}\left(H-\alpha H_{2}\right)-\alpha F_{\varepsilon_{Q}} H_{2}\right)\left(F_{Q}\left(H-\alpha H_{2}\right)-\alpha F_{\varepsilon_{Q}} H_{2}\right)^{*}$

$G_{\Delta}=\left(\begin{array}{l}G_{Q}\left[|H|^{2}+\alpha^{2}\left|H_{2}\right|^{2}-2 \alpha \operatorname{Re}\left(H H_{2}^{*}\right)\right] \\ +\alpha^{2} G_{\varepsilon_{Q}}\left|H_{2}\right|^{2}-2 \alpha \operatorname{Re}\left[F_{Q} F_{\varepsilon_{Q}}^{*}\left(H H_{2}^{*}-\alpha\left|H_{2}\right|^{2}\right)\right]\end{array}\right)$

Noting that $F_{\varepsilon_{Q}}=F_{\hat{Q}}-F_{Q}$ and $G_{\varepsilon_{Q}}=G_{Q}+G_{\hat{Q}}-2 \operatorname{Re}\left(F_{Q} F_{\hat{Q}}^{*}\right)$, then

$G_{\Delta}=|H|^{2} G_{Q}+\alpha^{2}\left|H_{2}\right|^{2} G_{\hat{Q}}-2 \alpha \operatorname{Re}\left[F_{Q} F_{\hat{Q}}^{*} H H_{2}^{*}\right]$

Consequently, the PSD of the remaining signal has a PSD depending upon 3 parameters:

- The knowledge of the equivalent filter response,

- The estimation of the incoming signal amplitude, and

- The estimation of the baseband signal.

The PSD of the original Galileo E1 signal entering the RA processing block is $G_{Q}|H|^{2}$. Consequently, the gain over the original signal can be quantified as:

$G_{D S P}=\frac{G_{\Delta}}{|H|^{2} G_{Q}}=1+\alpha \frac{\alpha\left|H_{2}\right|^{2} G_{\hat{Q}}-2 \operatorname{Re}\left[F_{Q} F_{\hat{Q}}^{*} H H_{2}^{*}\right]}{|H|^{2} G_{Q}}$

The PRS spreading code will be a very long aperiodic spreading code. Thus, it is possible to represent its PSD by the PSD envelope (by opposition to the peak spectrum of GPS C/A, for instance, due to the short periodic spreading sequence used). In this case, the $\hat{Q}$ will also have a very long aperiodic spreading code (with or without estimation errors). Consequently, the normalized PSDs of $Q$ and $\hat{Q}$ are the same.

The correlation between $Q$ and $\hat{Q}$ can be written as:

$$
E\left[Q \hat{Q}^{*}\right]=\int_{-\infty}^{+\infty} F_{Q} F_{\hat{Q}}^{*} d f
$$

However, because both $Q$ and $\hat{Q}$ have the same modulation, it means that their cross-spectrum will have the same PSD envelope. Consequently, for infinite random codes, $F_{Q} F_{\hat{Q}}^{*}=K G_{Q}$. And,

$$
E\left[Q \hat{Q}^{*}\right]=\int_{-\infty}^{+\infty} F_{Q} F_{\hat{Q}}^{*} d f=K \int_{-\infty}^{+\infty} G_{Q} d f=K E\left[Q Q^{*}\right]
$$

Thus $K=\frac{E\left[Q \hat{Q}^{*}\right]}{E[Q Q]}$

$E\left[Q \hat{Q}^{*}\right\rfloor$ will depend upon the probably that the spreading chips of $\hat{Q}$ were correctly estimated.

It has been seen that the probably of correctly estimating $Q$ was depending upon the sign of $W$.

If $W=1$, then

$$
\begin{aligned}
& E\left[Q \hat{Q}^{*}\right]_{W=1}=P(Q(i)=\hat{Q}(i)) q_{1}^{2}-P(Q(i) \neq \hat{Q}(i)) q_{1}^{2} \\
& E\left[Q \hat{Q}^{*}\right]_{W=1}=(1-P(Q(i) \neq \hat{Q}(i))) q_{1}^{2}-P(Q(i) \neq \hat{Q}(i)) q_{1}^{2} \\
& E\left[Q \hat{Q}^{*}\right]_{W=1}=q_{1}^{2}\left(1-2 P_{1}\right)
\end{aligned}
$$

where $P_{1}$ is the probability that the sign of the sample $c(i)$ is well estimated when $W=1$.

If $W=-1$, then

$$
E\left[Q \hat{Q}^{*}\right]_{W=-1}=q_{-1}^{2}\left(1-2 P_{-1}\right)
$$

Since the OS spreading sequence is a random sequence,

$$
\begin{aligned}
& P[W=1]=P[W=-1]=\frac{1}{2} . \text { Thus, } \\
& E\left[Q \hat{Q}^{*}\right]=\frac{1}{2} q_{1}^{2}\left(1-2 P_{1}\right)+\frac{1}{2} q_{-1}^{2}\left(1-2 P_{-1}\right)
\end{aligned}
$$

And finally, 
$K=\frac{q_{1}^{2}\left(1-2 P_{1}\right)+q_{-1}^{2}\left(1-2 P_{-1}\right)}{q_{1}^{2}+q_{-1}^{2}}$

This means that the reduction of the DSP level can be approximated by:

$G_{D S P}=1+\alpha \frac{\alpha\left|H_{2}\right|^{2}-2 K \operatorname{Re}\left[H H_{2}^{*}\right]}{|H|^{2}}$

Assuming that the filter response is perfectly known, it gives:

$G_{D S P}=\frac{\left.G_{\Delta}\right|_{H=H_{2}}}{|H|^{2} G_{Q}}=1+\alpha(\alpha-2 K)$

It is difficult to compute the theoretical value for $P_{1}$ and $P_{-1}$. However, it is possible to determine their value by simulations. The simulation parameters chosen were the same as the ones used to obtain Table 1. Moreover, only
$10 \mathrm{~ms}$ of signals were analyzed. This value was chosen to reduce the computational load, while ensuring that the underlying PRS spreading code was long enough (25575 chips) to mimic a smooth enough PSD. This was done extensively assuming that $\mathrm{H}=\mathrm{H}_{2}$. Several values were taken for the estimation error of the PRS amplitude $(\alpha)$.

Table 2 shows the results. It can be seen that the improvement brought by the proposed technique is significant, assuming that the Galileo E1 payload filter response is perfectly known, and for different values of the loss $L$ and of the bias of the Galileo E1 power estimate. In particular, simulations match very well the theory.

The results show that the higher the Galileo E1 signal strength, the better the proposed algorithm will be able to reduce the Galileo E1 PSD. This is important because, as already explained, it is the Galileo signals coming within the radio-telescope main lobes that will have a significant impact on the PFD values.

Table 2 - Example of PSD Gain Brought by the Proposed Technique for Different Losses and Different Biases for the Galileo E1 Power Estimate

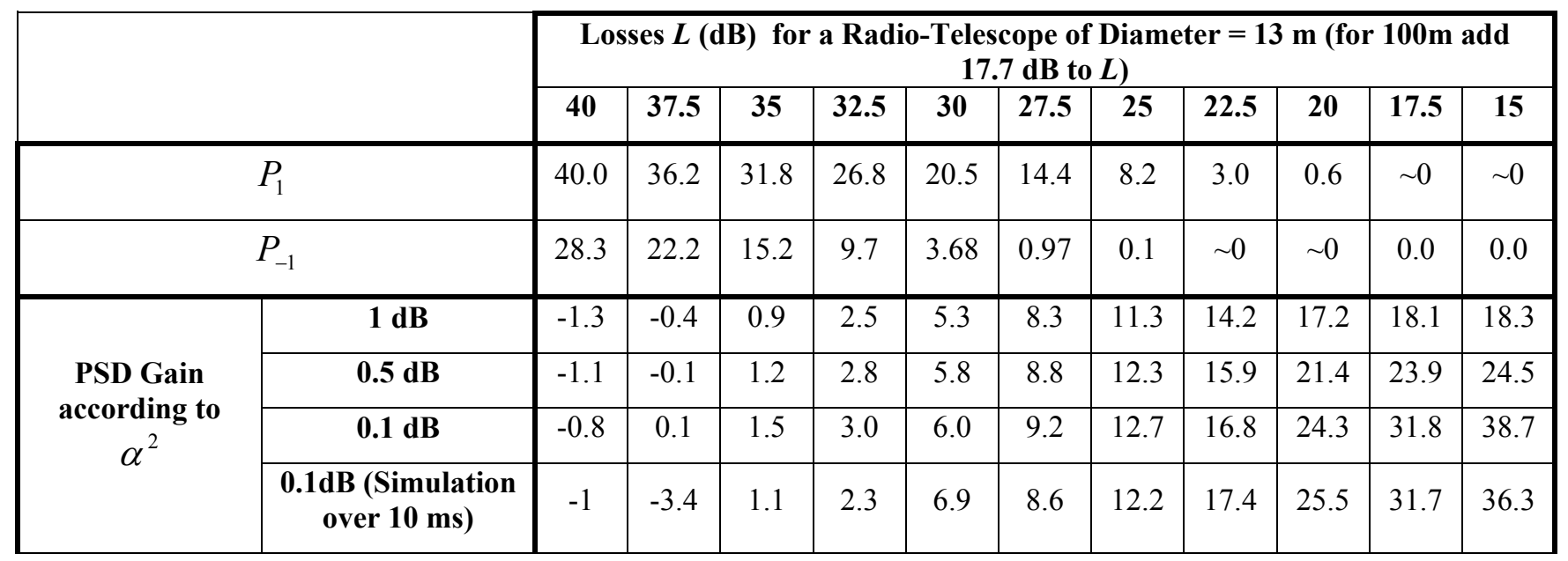

Reducing the PSD level of the interfering signal is equivalent to a reduction of the antenna gain in the direction of that satellite (Indeed, the PSD of the remaining signal is also a PRS PSD). Thus, this would mean that the method is equivalent to receiving the Galileo signal with a lower power. Figure 6 shows the resulting equivalent antenna gain taking into account the proposed method for the case where $\alpha^{2}=0.5 \mathrm{~dB}$.

Using this new equivalent gain pattern, it is then possible to re-run the simulations based on the ANFR program. The new results give a margin of approximately $10 \mathrm{dBs}$. This would mean that the Galileo E1 payload filter constraint could be relaxed by $10 \mathrm{dBs}$, which would be a significant step.

\section{Conclusions}

The first part of this paper showed the level of protection recommended by the ITU for the RA band 1610.6-1613.8 MHz. In particular, an emphasis is put on the impact of GLONASS on this band. Indeed, the first GLONASS frequency plan overlapped the RA band, created an intolerable level of interference for the RA users. The RA community and the Russian Federation then decided to enter a coordination process in order to set a series of agreements resulting, among other decisions, in a modification of the GLONASS frequency plan, as well as in a continuing collaboration. The latest outcome at the WRC 2007 was to exclude GLONASS from respecting 
the ITU recommendation on the RA band, based on the fulfillment of the IUCAF/Russian Federation agreement.

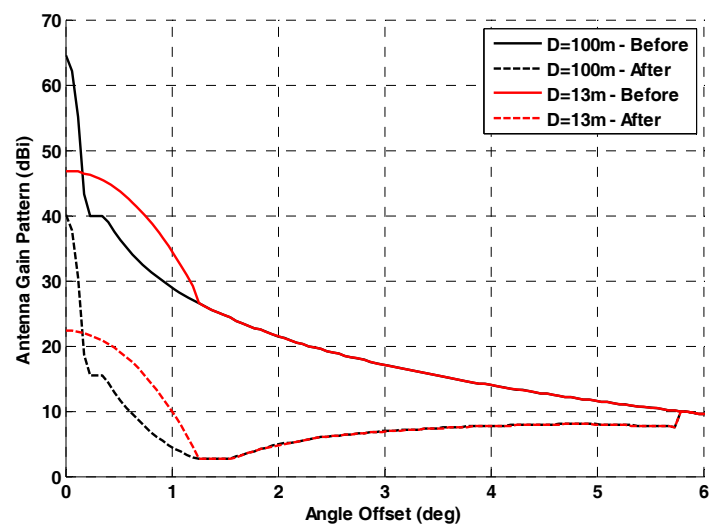

\section{Figure 6 - Equivalent Antenna Gain Pattern Before and After the Proposed Technique}

In a second part, the impact of the ITU recommendations on the future Galileo E1 signal was investigated. It was shown that respecting the recommendation means, for Galileo, to have a payload filter with a slope of approximately $37 \mathrm{dBs}$ in $15 \mathrm{MHz}$, which is a strong constraint, in particular for a satellite payload design, and given the presence of the Galileo E1 PRS at the edge of the Galileo transmitting band.

This paper then investigates a technique meant at removing a GNSS signal (based on CDMA) present in the RA band that could help either:

- to reduce the constraint on the GNSS payload filter, or

- to prevent strong interference from a GNSS that would be above the ITU recommendation.

It is based on the use of a dual structure: a "GNSS processing block" meant to estimate the baseband GNSS signal; and a "RA processing block" that first removes the estimated signal from the incoming signal and then processes the 'clean' RA signal.

This algorithm is deeply described in the context of Galileo E1. In this case, the main part of the algorithm concentres in estimated the PRS and the IM signals. The algorithm is then enhanced by forcing the estimated signal to have a cosine-phase $\mathrm{BOC}(15,2.5)$ modulation.

The analysis of the method showed that its success is based on many mainly 3 parameters:

- the accurate knowledge of the satellite payload filter (including outside the transmitted band), and the receiver filter,

- the accuracy of the estimation of the open signal amplitude,

- the robustness of the estimated PRS+IM signal.
As a first step, the performance results assumed that the Galileo E1 payload filter was perfectly known. With that in mind, it showed the proposed algorithm could potentially trigger a relaxation of the required filtering constraint in the RA band by approximately $10 \mathrm{dBs}$. This means that, still meeting the ITU recommendations for the RA band, Galileo satellites could use an E1 payload filter with a significantly lower slope and thus induce a more cost effective solution and a better service for the end user.

These results, though, should be looked at as preliminary results that will trigger further investigations. In particular, the RF design has to be studied. Also, it is crucial to know if it is possible to know well enough the payload filter outside its useful bandwidth to remove accurately the GNSS signal from the incoming RA signal. Finally, it is also necessary to estimate the losses occurring in the receiver front-end (cable losses, noise factor, etc...) in order to have a more accurate assessment of the method.

\section{BIBLIOGRAPHY}

ANFR, Unwanted Emission Levels from Galileo in the Band 1610.6-1613.8 MHz (Radioastronomy), Report

Bunton, J.D. (2000), Cancellation of GLONASS Precision Code from Cross Correlations, Technology Pathways to the Square Kilometre Array, Jodrell Bank UK.

Briggs, F.H, J.F. Bell and M.J. Kesteven (2000), Removing Radio Interference from Contaminated Astronomical Spectra using an Independent Reference Signal and Closure Relations, The Astronomical Journal, pp. 3351 - 3361.

Cohen, R.J. (2002), GLONASS and Radio Astronomy, Proceedings of the IUCAF Summer School, Green Bank, West Virginia, June 9-14, pp. 158-170

Ellingson, S. W., J. D. Bunton, and J. F. Bell (2001), Removal of The GLONASS C/A Signal from $O H$ Spectral Line Observations using a Parametric Modeling Technique, The Astrophysical Journal Supplement Series, July, pp. 87-93

Ellingson, S.W., and G.A. Hampson (2003), Mitigation of Radar Interference in the L-Band Radio Astronomy, The Astrophysical Journal Supplement Series, July, pp. 167-176

ERC (1997), European Radiocommunications Committee Report on Interference calculations from MSS Satellites into Radio Astronomy Observations.

Galileo OS ICD (2008), Galileo Open Service - Signal in Space - Interface Control Document-Draft 1.

Galt, J. (1991), Interference with Astronomical Observations of OH Masers from the Soviet Union's GLONASS Satellites, in Astronomical Society of 
Pacific Conference Series on Light Pollution, Radio Interference, and Space Debris, Vol. 17, IAU Colloquium 112, D.L. Crawford, Ed., p. 213

Hein, G., J.-A. Avila-Rodriguez, S. Wallner, A R. Pratt, J Owen, J-L Issler, J W. Betz, C. J. Hegarty, Lt S. Lenahan, J. J. Rushanan, A. L. Kraay, and T. A. Stansell (2007), MBOC: The New Optimized Spreading Modulation Recommended for GALILEO L1 OS and GPS L1C, Proceedings of IEEE/ION PLANS 2006, 2427 April 2006, San Diego, CA, USA.

Jeffs, B.D., L. Li, and K.F. Warnick (2005), Auxiliary Antenna-Assisted Interference Mitigation for Radio Astronomy Arrays, IEEE Transactions on Signal Processing, Vol. 53, No. 2, February, pp.439-451.

Leshem, A, and A-J Van Der Veen (1999), Introduction to Interference Mitigation Techniques in Radio Astronomy, Perspectives on Radio Astronomy Technologies for Large Antenna Arrays, Netherlands Foundation for Research in Astronomy

Leshem, A, and A-J Van Der Veen (2000), Multichannel Interference Mitigation Techniques in Radio Astronomy, The Astrophysical Journal Supplement Series, November, pp. 355-376

Lestarquit, L., L. Ries, J. Dantepal, C. Zanchi, A. Mallet, and P. Dumon (2001), GNSS2 Payload: Propagation Delays; Possible Architectures, Proceedings of the Institute of Navigation GPS, Salt Lake City, Utah, USA

Monstein C., and H. Meyer (2007), RFI in Radio Astronomy Band $1610.6 \mathrm{MHz}-1613.8 \mathrm{MHz}$ at Bleien Observatory, Physics, Astronomy and Electronics Work Bench.

Ohishi, M. (2008), WRC 2007 Results, Powerpoint Presentation, RAFCAP Meeting 2008

Poulsen, A. (2003), Real-Time adaptive Cancellation of Satellite Interference in Radio Astronomy, Master of Science, Brigham Young University

Rebeyrol, E. (2007), Galileo Signals and Payload Optimization, $\mathrm{PhD}$ Thesis 